\title{
100W, Fully-Fiberised Ytterbium Doped Master Oscillator Power Amplifier Incorporating Adaptive Pulse Shaping
}

\author{
Dejiao Lin, Shaif-ul Alam, Kangkang Chen, Andrew Malinowski, Steve Norman* and David Richardson \\ Optoelectronics Research Centre, University of Southampton, Southampton, SO17 1BJ, United Kingdom \\ * SPI Lasers UK Ltd., Wellington Park, Hedge End, Southampton, SO30 2QU, United Kingdom \\ dxl@orc.soton.ac.uk

\begin{abstract}
We report a pulsed, fully-fiberised, Yb-doped MOPA with a maximum average output power of $100 \mathrm{~W}$. Adaptive pulse shaping was incorporated to reduce the impact of nonlinearities, delivering $2 \mathrm{~mJ}$ flat-topped pulses with $20 \mathrm{~kW}$ peak power.

(C) 2009 Optical Society of America

OCIS codes: (060.2320) Fiber Optics Amplifiers and Oscillators; (320.5540) Pulse shaping
\end{abstract}

\section{Introduction}

Pulsed fiber MOPAs have received increasing attention over the past decade offering potential advantages in terms of efficiency, compactness, reliability, ease of thermal management and high average power levels relative to existing pulsed laser approaches [1]. For practical reasons it is highly desirable to work with fully-fiberized MOPA systems. However, to date, most previously reported pulsed MOPAs operating at average powers of a few 10's of Watts have incorporated free-space, end-coupling of the pump radiation.

Here we present an all-fiber, nanosecond pulsed $\mathrm{Yb}$-doped MOPA without any free space pumping. Average output powers as high as $100 \mathrm{~W}$ were obtained at $69 \%$ slope efficiency with respect to launched pump power. Using pulse shaping technology we obtain square shaped output pulses, minimizing the pulse peak power for a given pulse duration and energy and thereby limiting the deleterious effect of nonlinearity - in particular Stimulated Raman Scattering (SRS) [2].

2. System setup and experimental results

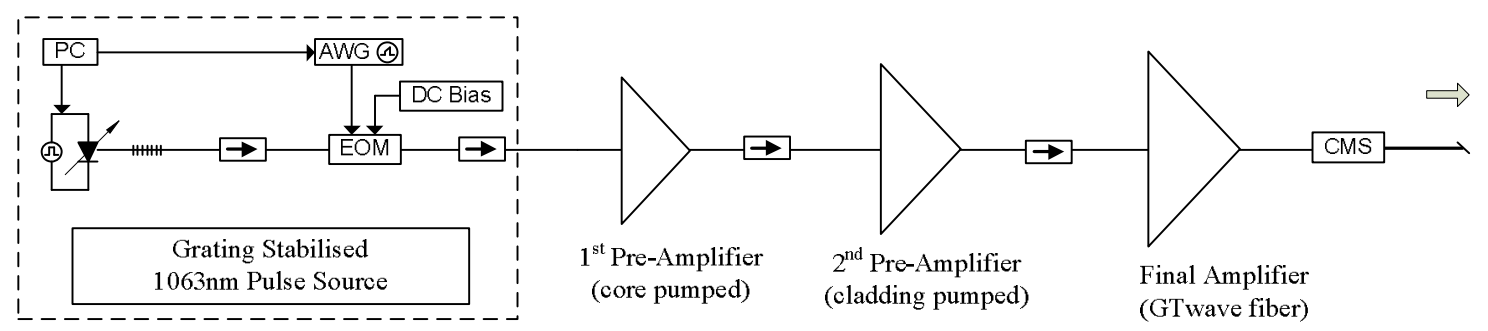

Fig. 1. The layout of the all-fiber Yb-doped MOPA. CMS: Cladding Mode Stripper

A schematic of the all-fiber Yb doped MOPA is illustrated in Fig. 1. The seed is a fiber pigtailed Fabry-Perot laser diode.The center wavelength of the seed signal is set to $1063 \mathrm{~nm}$ by an external PM fiber Bragg grating. The directly modulated optical output of the seed laser was pre-shaped using an electro-optic modulator (EOM) driven by an Arbitrary Waveform Generator (AWG) with 4ns resolution. The pulse width of the EOM output was set at 100ns at a repetition rate of $50 \mathrm{kHz}$.

Around $0.1 \mathrm{~mW}$ of average output power from the EOM was amplified by $60 \mathrm{~dB}$ using a three-stage amplifier chain. The first pre-amplifier stage was based on a core pumped, $\mathrm{Yb}^{3+}$-doped active fiber (fabricated in-house) with a core diameter and NA of $6 \mu \mathrm{m}$ and 0.12 respectively. The amplifier was bi-directionally pumped with $976 \mathrm{~nm}$ single-mode laser diodes. A maximum saturated output power of $30 \mathrm{~mW}$ was obtained from this stage. The output of the first stage amplifier was coupled into the second pre-amplifier stage via an in-line optical isolator. The active medium of the second-stage amplifier was a double-clad, $\mathrm{Yb}^{3+}$-doped fiber with a core diameter and NA of $6 \mu \mathrm{m}$ and 0.13 respectively. The cladding diameter of the polymer coated, petal-shaped fiber was $130 \mu \mathrm{m}$ with pump guiding NA of 0.45 . The gain medium was co-pumped by $915 \mathrm{~nm}$ fiber-pigtailed broad-stripe diode lasers through a $(6+1)$ tapered fiber bundle (TFB). The maximum saturated output power from this stage was $1.8 \mathrm{~W}$.

The power amplifier comprised a $6.7 \mathrm{~m}$ long Yb-doped, SPI proprietary, non-photodarkening, large mode area (LMA) GTWave ${ }^{\mathrm{TM}}$ [3] fiber. The GTWave fiber facilitates pump coupling to the doped fiber without using its ends. The amplifier was bi-directionally pumped using 940nm fiber pigtailed broad-stripe laser diodes. 
A maximum average output power of $100 \mathrm{~W}$ was obtained at a launched pump power of $149 \mathrm{~W}$ corresponding to a slope efficiency of $69 \%$ as plotted in Fig. 2. When a square input pulse was coupled into the MOPA chain, the leading edge of the optical pulse was amplified preferentially over the trailing edge (shown in Fig. 3), leading to distortion of the final output pulse. The instantaneous power of the leading edge could be several times higher than the rest of the pulse envelope resulting in undesirable SRS generation and limiting the maximum pulse energy extraction from the system before the onset of nonlinear effects or damage. A square output pulse form is desirable to scale-up the pulse energy since it provides for the lowest pulse peak power thereby minimizing deleterious nonlinear effects. Fig. 3 shows the square shaped output pulses obtained while operating the MOPA at various output powers while the inset shows the corresponding input pulse profiles. The exponential rising edge from the leading to the trailing edges of the input pulses increased with increasing output powers from the power amplifier. The spectra of the shaped signal pulses for various output powers are shown in Fig. 4. It is observed that the spectral bandwidth increases with an increase in output power due to self-phase modulation (SPM), however no sign of SRS was observed even when the MOPA was running at the maximum average output power of $100 \mathrm{~W}$ (with a corresponding peak power of $20 \mathrm{~kW}$.) Further scaling in output power and hence the pulse energy was primarily limited by the available pump power. No degradation in output power was observed during the experimental work which highlights the importance of using non-photodarkening fiber in the power amplifier stage.

The output beam quality $\left(\mathrm{M}^{2}\right)$ was varied between 2 to 3 depending on the choice of the beam delivery fiber spliced to the output fiber. Detailed $\mathrm{M}^{2}$ measurement data will be presented at the conference.

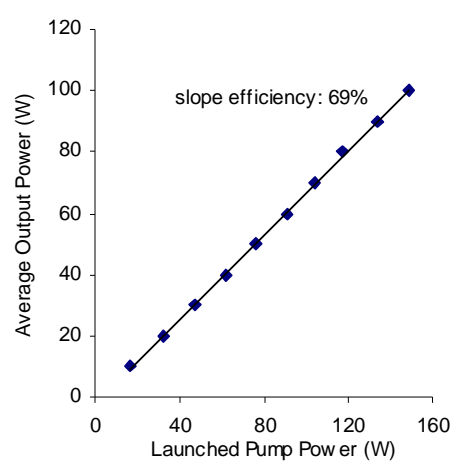

Fig. 2. Efficiency plot of $1063 \mathrm{~nm}$ signal

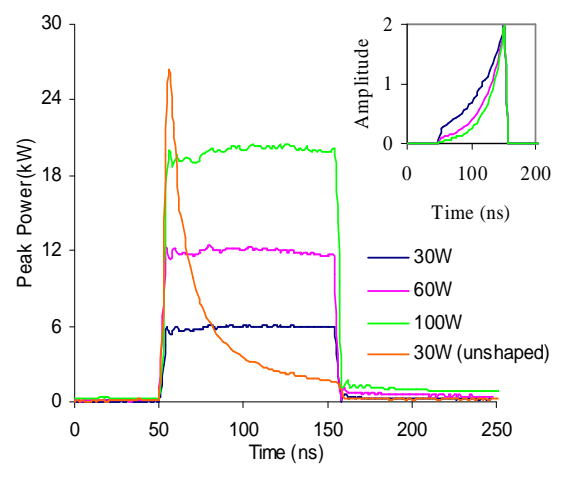

Fig. 3. Temporal plots of the optical pulses

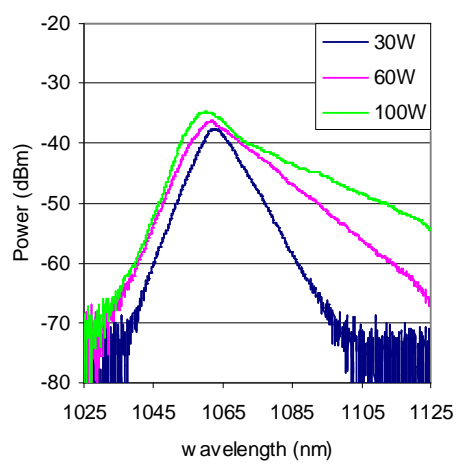

Fig. 4. Spectra of the signal output

\section{Conclusions}

We have successfully demonstrated an all-fiber, Yb-doped MOPA. A maximum average output power of 100W at an overall slope efficiency of $69 \%$ was achieved from the final stage amplifier. Using adaptive pulse shaping of the seed laser we successfully reduced the impact of dynamic gain saturation and optical Kerr/Raman nonlinearities within the MOPA. Pulse energies of $2 \mathrm{~mJ}$ and peak powers as high as $20 \mathrm{~kW}$ were achieved in this work. Such high energy, high peak power pulses can be used for a wide range of applications including rapid prototyping, material processing, cutting and dicing of silicon wafers, micromachining and marking to name but a few.

\section{Acknowledgement}

This work was supported in part by the UK Technology Strategy Board project HEGAC. The non-photodarkening GTWave fiber used in the final amplifier was provided by SPI Lasers UK Ltd.

\section{References}

[1] A. Piper, A. Malinowski, K. Furusawa, and D. Richardson, "High-power, high-brightness, mJ Q-switched ytterbium-doped fibre laser," Electronics Letters, 40, 928-929 (2004).

[2] Y. Wang and H. Po, "Dynamic characteristics of double-clad fiber amplifiers for highpower pulse amplification," Journal of Lightwave Technology, 21, 2262-70 (2003).

[3] C. Codemard, K. Yla-Jarkko, J. Singleton, P.W. Turner, 1. Godfrey, S.-U. Alam, J. Nilsson, J. Sahu and A. B. Grudinin, "Low noise, intelligent cladding pumped L-band EDFA", ECOC 2002, Copenhagen, Denmark, Sept. 2002, PD1.7. 